compatible with the preservation of health, but the accommodating powers of the system were limited, and it would be a great gain if by united efforts something like definite knowledge of such limits could be approached, so as to be able to predicate of a given locality that it would be favourable or inimical to health-that it would mitigate or intensify certain forms of disease. Geological formation had also its influence, by no means inconsiderable, on health and disease, not merely in so far as it might facilitate drainage, etc.; its more immediate effects would depend upon the character of the strata of any given locality, and on the water-supply thence derived. The full importance of water as a modifying element of climatic influences, and as a powerful agent ever present and participating in all physiological action and in every pathological change, was not by any means yet appreciated. He was speaking of water in its natural state, and free from those pollutions which were unhappily common. The members were familiar with some of the effects due to water per se, such as the prevalence of stone in certain districts and of goitre in others; but there were many other not so strongly pronounced manifestations of which they were entirely ignorant, or possessed but a vague and uncertain knowledge.

He next spoke of another matter requiring investigation, viz., the facility with which certain water not charged with lime-salts acted upon metals, acquiring thereby highly deleterious properties through passing through lead pipes : a danger not yet fully recognised as a source of disease, but which, either slowly and insidiously, or more rapidly, effected its poisonous work. Inquiry in regard to this matter was still more necessary in the present day from the circumstance, that from some cause, probably from a difference in the mode of manufacture, the action of the water on the lead was far more energetic than in former periods. Then, there was the prevalent use of copper vessels for culinary purposes, the baneful effects of which had been witnessed by himself in not a few instances. Other metals had their own peculiar dangers, and a new element in the genesis and modification of disease had been recently introduced with the extensive use of galvanised iron for the conveyance and storage of water in certain districts, a train of evil-marked symptoms ensuing upon the daily use of water thus contaminated. Not yet, too, was there by any means a full comprehension of the slow, silent, and disastrous consequences of the habitual use of adulterated food and drink, of the very lights burnt, and, as regarded certain colours, of the very clothes worn. Rooms were decorated with papers whose delicate and beauteous hues charmed the eye, while there was effluent from them a deadly poison. It had yet to be learned how much of chronic ill-health was due to these respective causes, and how far change of air owed its salubrious effects to an escape from these noxious influences.

Having at length dwelt upon the great gain which would come to medical science if minute and remote causes of disease were traced out by the machinery he had indicated, he referred to the action of stimulants as a subject deserving of consideration, with a view to the acquisition of more definite knowledge upon it. The diversity of opinion that obtained in reference to the medicinal and dietetic use of these, and the discrepancy of practice that prevailed, were greatly to be deplored, inasmuch as they imparted to the medical art a gratuitous measure of uncertainty, and detracted from its scientific pretensions. A wide latitude of opinion might be expected to prevail, but it ought not to be so wide as to embrace views so conflicting and irreconcilable that their existence would seem to indicate that a blind empiricism rather than a rational method was the guide of their practice. He referred to the investigations of Professor Parkes and others, as adding much to the elucidation of this subject.

In conclusion, he said if the collective efforts of the Association could be from time to time concentrated on subjects of inquiry similar to those he had mentioned, under some well devised methods of observation, they might well hope for the elucidation of much that was now vague and uncertain. The supplementary reports of the Privy Council and Local Government Board just issued demanded the careful perusal of every member of their profession, as well as of every thoughtful and humane citizen, for they revealed facts at which humanity stood aghast and decency was put to open shame. Their chief burden was the production of diseases by filth, and Mr. Simon said that the deaths in each year registered in this country (now about half a million a year) were fully 125,000 more numerous than they would be if existing knowledge of the chief causes of diseases affecting masses of population were reasonably well applied throughout England, this being the conviction of persons who had studied the subject. And with such terrible power did these preventable causes of disease in certain districts impress their fatal influence on infant life, that Mr. Simon had felt it to be consistent with truth to apply to such districts the epithet "Herodian"- - a term expressive of the sacrifice of the weak, the helpless, and the innocent. "Put not your trust in princes", sang David in olden times, and to this injunction, as regarded sanitary measures, they might now add, and still less in Governments, struck with apathy and palsied with indifference, who stood feebly tottering in the presence of "vested interests", and the "liberty of the subject", piously ejaculating, with averted eyes and uplifted hands, "Sanitas sanitatum, omnia sanitas". He exhorted the Branch, as members of a noble profession, as philanthropists, and as citizens, to bring all their highest mental powers, their best energies, their fervent resolves, to aid in a holy warfare against all preventable diseases. Ten thousand hands could not be ready, hearts willing, and voices raised, all in vain. They might not achieve a victory that should win the applause of a senate or the shouts of a people, or secure for themselves individually social distinction or titular agyrandisement; but there would remain for them the quiet enjoyment that flowed from a sense of a duty performed, a mission fulfilled, a conscience satisfieda satisfaction that no patent could confer, no fountain of honour bestow.

\section{ON THE PRODUCTION OF GLYCOSURIA BY THE EFFECT OF OXYGENATED BLOOD ON THE LIVER.*}

By F. W. PAVY, M.D., F.R.S.,

Physician to and Lecturer on Physiology at Guy's Hospital.

THE author referred to a former communication to the Royal Society on "Lesions of the Nervous System producing Diabetes" (Proceedings of the Royal Society, vol. $x, 1859-60)$, in which he made known that division of certain parts of the sympathetic system occasioned the presence of sugar in the urine. The results given in that paper showed that there were other means besides Bernard's celebrated experiment of puncturing the floor of the fourth ventricle, by which artificial diabetes could be induced, but they did not explain the reason of the appearance of the sugar, and the author still sought to discover something upon this point. The inquiry was pushed in various directions, but always with a fruitless issue until the summer of 1874 . The author for some time past has been led to look to an altered condition of the blood flowing to the liver as likely to prove the most probable cause of the transformation of amyloid substance into sugar, which evidently constitutes the foundation of the artificial diabetes following operations on the nervous system. Schiff is of this view, and (Fournal de l'Anatomie et de la Physiologie, Paris, 1866 ) has referred the escape of sugar from the liver, and thence the production of glycosuria, to the development of a ferment in the blood as a result of the hyperæmia (not necessarily of the liver) which follows the operations on the nervous system which occasion artificial diabetes ; but, although the author has carefully examined this opinion, he cannot obtain evidence of the development of a ferment in the manner asserted. He has further tried the effect of introducing a secretion, viz, saliva, into the circulatory system, which is known to act as an energetic ferment upon the amyloid substance of the liver, and on one occasion he found, from some cause or other, that the urine became, to a moderate extent, saccharine, but in a large number of other experiments the operation was attended with a negative result.

Having so far proceeded without success, it occurred to the author to try the effect of introducing defibrinated arterial blood into the portal system. He was led to experiment in this way from having some time previously observed that when arterial blood only was allowed to flow through the liver, as, for instance, when the portal vein was tied and the hepatic artery left free, sugar escaped from the organ to such an extent as to render the contents of the circulatory system strongly saccharine. This result he had commented upon as being somewhat surprising, and as furnishing evidence standing in opposition to Bernard's glycogenic theory. He had not succeeded by the operation in producing glycosuria, because, as it appeared to him, no urine was secreted, owing to the ligature of the portal vein leading to such a diversion of blood from the general circulation by the accumulation occurring in the portal system, that the flow through the kidney was too slight to allow of it. He had endeavoured to overcome this obstacle by connecting, through the medium of a cannula, the portal with the right renal vein after ligaturing the corresponding renal artery. If the experiment had succeeded, the liver would have been left with its arterial supply, but the portal stream would have been diverted and made to reach the inferior cava without traversing the hepatic vessels.

As regards the operation, this the author found he could accomplish, but each time he performed the experiment the object he had in view was frustrated by the cannula becoming quickly filled with a plug of

* Abstract of a paper read at the Royal Society, June 17 th, 1875 . 
blood-clot. It was whilst under this difficulty that the thought of collecting blood from an artery, defibrinating it, and then introducing it into the portal system, occurred to him. He had considered it possible that some slight effect might be perceptible, but had not anticipated the strongly marked result which is producible. The amount of blood used has been from ten to eighteen fluid ounces. After the production of anæsthesia by chloroform, the blood was collected from the carotid artery, stirred in order to defibrinate it, strained, and then very slowly injected into a branch of the mesenteric vein. In one experiment, where half an hour had been employed in making the injection, the urine at the completion of the operation contained a notable amount of sugar, and half an hour later showed by analysis the presence of fifteen grains to the fluid ounce. In a second, the urine contained ten, and in a third fourteen grains to the fluid ounce when collected three quarters of an hour after the operation. The experiments were performed upon dogs, and in each case it had been ascertained that the urine was clevoid of sugar before the operation.

Having noticed the effect which have been described from the injection of oxygenated blood into the portal system, it became necessary to ascertain positively that it was attributable to the oxygenated condition of the blood, and not to any other cause. To decide this point, an appeal to the counterpart experiment was made. Defibrinated venous instead of arterial blood was injected into a branch of the mesenteric vein, and upon each occasion where such an operation has been performed a negative result has been obtained.

With the evidence thus furnished, the conclusion may be warrantably drawn, that oxygenated blood in some manner influences the liver so as to lead to the production of glycosuria. It may be inferred that, contrary to the effect of venous blood, it promotes the transformation of amyloid substance into sugar. The suggestion naturally occurs, that what has been stated above affords an explanation of the glycosuria occurring after Bernard's puncture of the fourth ventricle and the various desions of the sympathetic. Without any new agent being called in, sufficient is presented in the state of the blood to account for the production of sugar that occurs. By a vaso-motor paralysis affecting the vessels of the chylopoietic viscera, the blood will reach the portal system without having become dearterialised in its natural way, and in this state it has been shown by the experiments narrated to possess the property of acting within the liver in such a manner as to determine the production of glycosuria.

\section{STATISTICS OF FEVER, ENDING IN RECOVERY AND IN DEATH, OCCURRING IN TWO THOUSAND CONSECUTIVE CASES OF LABOUR.*}

By I. HARRINSON, F.R.C.S.,

Fellow of the Obstetrical Society of London.

IN making my contribution to the now all-discussed subject of puerperal fever, I propose to do so from, as far as possible, a practical point of view ; to follow the example so admirably set on the introduction of the subject at our last meeting. By practical, I mean as the cases occurred in practice, with all their, it may be, obscurity of origin, irregularity of progress, and, in some cases, unlooked for terminations. I shall not attempt to make things fit where there was no fitness; much less shall I draw a line, hard and fast, in their causes, symptoms, pathology or treatment. I shall give the cases as they were written at the time of their occurrence, with the remarks, if any, appended at the time, and draw inferences from them fairly apparent to you and to me. It cannot be expected that the earlier cases would be examined as closely and with the same light as the latter ones, but, as far as they go, they may be taken as truthful representations.

I need not insist in this place on the normal abnormality of a puerperal woman, specially on her system teeming with the effete products of an involuting uterus and the waste of parturient muscular effort, oppressed with the diverted stream of foetal nutrition, and charged with the detritus of an obsolete circulation, to which kidney implication may be added; nor will I insist on her susceptibilities, increased as they must ever be by the accidents of hæmorrhage, unhealthy surroundings, and mental conditions. It is a state sui generis, and only has a parallel, as Dr. Ord remarked to me a few days ago, in a system saturated with the material of gout. It is a state which renders her specially vulnerable to toxæmic impressions.

A good illustration of toxic susceptibility, on a grand scale, is afforded

$$
\text { * Read before the Pathological Society of Reading. }
$$

at the present time by our new Pacific acquisition. There, the virgin blood of the Fijians, unacclimatised to zymotic influences, unprotected by previous attacks, and unprepared for any, is at once disordered by the invasion of the weakest of European foes-that of measles-which revels in its freedom from antagonism, and now is numbering its victims by the thousand.

I will take it for granted that puerperal fever is admitted to be a toxæmia, the septinous matter being introduced by contagion (contact) or by infection (any and every other mode of introduction). It is possible that this septinous material may be inoculated before labour; and it is pretty certain that infection, as of scarlatina or erysipelas, may be received at an indefinite time during pregnancy, and only develope itself after delivery.

I will not attempt to draw a line between the access, progress, terminations, lesions, etc., of the specific poisons, as scarlatina, erysipelas, and those induced by animal matter, fresh as after a post mortem examination, or more or less putrid, as from a dissecting-room, though I believe it is generally admitted that the fresher the subject greater the danger; or those arising from a bit of retained placenta, or a nonexpelled clot; between differences in kind and differences in degree. Undoubtedly there are differences, and each cause produces a different series of effects, which only require to be carefully classified to establish a differential diagnosis. This is the work yet to be done. It is possible, also, that a practitioner, having met with a number of similar cases dependent on the same cause or causes, as in one hospital or locality, might be led to believe that there was one special puerperal fever.

It is proved by the experiments of Dr. Richardson that septinous poisons may be diluted with water to a given extent, and yet remain active up to an extreme of dilution. This fact warns us how the smallest quantity of morbid matter may inoculate a puerperal woman. $\mathrm{HI}$ proves, also, that the intensity of the poisonous matter is increased in the most marked degree, and in a manner that seems to defy computation, with the transmission of the poison through fresh series of animals. This fact explains why, when a practitioner has a succession of unfortunate cases, particularly if he redouble his attentions to each, the last cases may be and are the worst.

It is reasonable to suppose that the risk both of contagion and of infection will be greater in some cases than in others, in the specific than in the non-specific ; for example, in those arising from the inoculation of erysipelas than in that produced by the mere non-expulsion of a clot.

"It appears to us", says the Lancet, " that the history of puerperal fever proves that the disease can be communicated without contact in some cases, and by contact in all. It is not essential that the disease should be taken from one patient to the other; the original contamination of the fingers may give rise to the disease in a series of consecutive cases. I cannot decide how, when a septinous material is inoculated, the effects ensue ; whether directly or whether by first inducing inflammation, from the product of which the virus is derived."

I may here state that to the more immediate effects of the introduction of septinous matter, either by contagion or infection, I limit the term septicæmia. Pyæmia is a secondary, or rather a tertiary, state, the product of phlebitis - one of the sequelæ of septicæmia. Blocking of the arteries-pulmonary, cerebral-by clots, local and migratory, must also be included among the dependencies of poisoned blood.

It is not easy to determine whether the dire effects of septinous poisons, seen in some instances, be dependent on the intensity of the poison, on the quantity introduced, or on the susceptibility of the individual to poison or to shock. Mr. Davis related, a few weeks ago, the case of a woman who died in half an hour from the sting of a hornet. It is well known what a very small amount of hæmorrhage from the uterus proves fatal in some cases. The greater protective power of four vaccine vesicles over one, demonstrates that quantity has some influence; some people catch everything; others take nothing. Practically, identity of cause does not produce uniformity of effect. Bear in mind Mr. Jeston's cases. A young woman nursed her father with erysipelas. She was seven months advanced in pregnancy. Two of her children had erysipelas, and, lastly, she was seized. Premature labour came on; puerperal fever supervened, and death occurred in seven days. The nurse who attended this woman was immediately afterwards seized with acute rheumatism and pericarditis, and died in seven days.

Among the things to be remembered in cases of puerperal fever are, that, though the origin may be erysipelas or scarlatina, it does not follow there should be the ordinary expression of either on the patient, nor does it follow that either the practitioner or the nurse introduced it. The patient may have been exposed unconsciously or recklessly to the poison of either, which, during her pregnancy, may have remained in a state of quiescence. A curious question arises, whether a woman in this state may not poison the fingers of the medical attendant at 